phase reactants, where there may be differences in the perception of efficacy. This perception of efficacy should be contrasted with scientific evidence

Acknowledgement: This study was funded by Bristol-Myers Squibb. Disclosure of Interests: Loreto Carmona Grant/research support from: Abbvie, Actelion, Astellas, BMS, Eisay, Gebro Pharma, Grünenthal, Leo Pharma, Lilly, MSD, Novartis, Pfizer, Roche, Sanofi-Aventis and UCB Pharma, Paid instructor for: Novartis, J. Narváez Consultant for: BristolMyers Squibb, Jaime Calvo Consultant for: Bristol-Myers Squibb, Janssen, Celgene, Sanofi Genzyme, Speakers bureau: Bristol-Myers Squibb, Alejandro Escudero Contreras: None declared, Santiago Muñoz Fernandez: None declared, José M. Rodríguez-Heredia: None declared, Susana Romero-Yuste: None declared, Paloma Vela Casasempere: None declared, Jose Luis Baquero Grant/research support from: MS, Abbvie, Roche, Becton Dickinson and General Electric, Employee of: Wyeth (10 years ago), Sara Lujan Valdés Employee of: Full time employee at Bristol-Myers Squibb, Juan J Sancho Jiménez Employee of: Full time employee at Bristol-Myers Squibb

DOI: 10.1136/annrheumdis-2019-eular.6883

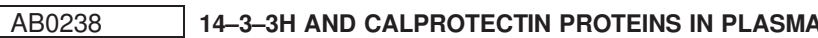 AS NEW BIOMARKERS IN EVALUATION OF THE ACTIVITY IN PATIENT WITH RHEUMATOID ARTHRITIS}

Anastasia Dmitruk $^{1}$, Stanislava Benevolenskaya ${ }^{1}$, Olga Karlina ${ }^{2}$, Dmitriy Vasilyev ${ }^{2}$ Nataliya Klyaus ${ }^{1}$, Dariya Kozlova ${ }^{2}$, Alexey Maslyanskiy ${ }^{1} .{ }^{1}$ Federal State Budgetary Institution "Almazov National Medical Research Centre" of the Ministry of Health of the Russian Federation, Saint Petersburg, Russian Federation; ${ }^{2}$ LLC "Sceintific and Production Firm "Abris+", Saint Petersburg, Russian Federation

Background: The determination of rheumatoid arthritis (RA) activity remains an important clinical task nowadays and it is a long and difficult process. There is no single approach to assess the activity of RA. The gold standard for evaluating RA activity is composite indexes, for example, DAS28. However, composite indices are not reliable in evaluation of the disease activity in patients with RA, as having a subjectity assessment. New biomarkers that reflect the immune inflammatory process in joints in RA is of great scientific importance. 14-3-3n and calprotectin markers in plasma are considered as promissing.

Objectives: To assess the value of $14-3-3 \eta$ and calprotectin in plasma as additional markers in RA activity determination.

Methods: 48 patients were examined with reliable diagnosis of RA, according to the ACR 2010 criteria. The mean age was 59 years, mean duration of the disease was 12.8 years. 4 patients in the group were in remission, 2 had low degree of RA activity, 20 - moderate, 22 - high. RA activity was determined according to the DAS28 ESR index. The concentrations of $14-3-3 \eta$ and calprotectin in the blood plasma were determined by ELISA (LLC «SPF «Abris+»). The obtained results were compared with laboratory (ESR, CRP) and clinical (tender joint count (TJC), swollen joint count (SJC), VAS, DAS28) parameters of RA activity. Results: $14-3-3 \eta$ and calprotectin proteins in plasma and ESR highly correlated with DAS28 ( $r=0.84 ; r=0.65 ; r=0.53)$, while CRP had moderate correlation with RA activity $(r=0.34)$. 14-3-3 $\eta$ and calprotectin proteins had high correlation with TJC ( $r=0.68 ; r=0.61)$ and moderate correlation with SJC $(r=0.56 ; r=0.41)$; ESR and CRP had weaker correlation ( $r=0.15 ; r=0.13 ; r=0.02 ; r=0.08)$ respectively.

The ROC analysis showed that sensitivity, specificity, and diagnostic accuracy of high RA activity determination are significantly higher for 14$3-3 \eta(A U C=0.98, p<0.001)$ and calprotectin $(A \cup C=0.89, p<0.001)$ than for $E S R$ ( $A \cup C=0.81, p<0.001$ ) and $C R P$ ( $A \cup C=0.72, p$ $<0.001$ ). The proteins $14-3-3 \eta$ and calprotectin reflect the relationship with the SJC (AUC $=0.89, p<0.0001 ; A \cup C=0.79, p=0.002$ ) with higher diagnostic accuracy, sensitivity and specificity, comparing with ESR (AUC $=0.63, p=0.19$ ) and CRP (AUC = 0.51, $p=0.88$ ).

It was also found, that calprotectin ( $A \cup C=0.8, p<0.001$ ) and 14-3-3n (AUC $=0.79, p<0.001)$ had greater sensitivity and specificity, as well as diagnostic accuracy, in TJC determination, compared with ESR (AUC = $0.57, p=0.4$ ) and CRP ( $A U C=0.52, p=0.8$ ). Determining the relationship between biomarkers and the patient's subjective assessment of RA activity (VAS), significant difference between 14-3-3n (AUC $=0.68, p=$ 0.06 ), calprotectin ( $A \cup C=0.63, p=0,16)$, ESR ( $\mathrm{AUC}=0.64, p=$ 0.14 ) and CRP (AUC $=056, p=0.57$ ) was not detected.

Conclusion: $14-3-3 \eta$ and calprotectin proteins in plasma are new promising biomarkers of immunoinflammatory process in joints in RA assessment, because they are more sensitive and specific markers of the disease activity, than conventional acute-phase proteins ESR and CRP. Disclosure of Interests: None declared

DOI: 10.1136/annrheumdis-2019-eular.5587

\section{AB0239 EFFECT OF SMOKING ON THE EFFICACY OF TNF INHIBITORS IN THE TREATMENT OF CHRONIC INFLAMMATORY IMMUNE-MEDIATED DISEASES: SYSTEMATIC REVIEW AND META-ANALYSIS}

Raquel Dos Santos Sobrín ${ }^{1}$, Alejandro Souto Vilas ${ }^{1}$, Jose Ramón Maneiro Fernández ${ }^{2}$, Antonio Mera Varela ${ }^{1}$, Juan J. Gómez-Reino ${ }^{3} .{ }^{1}$ Clinical University Hospital of Santiago de Compostela, Rheumatology Department, Santiago de Compostela, Spain; ${ }^{2}$ Hospital Complex of Pontevedra, Rheumatology Department, Pontevedra, Spain; ${ }^{3}$ Instituto de Investigación Sanitaria - Hospital Clínico Universitario de Santiago (IDIS), Experimental and Observational Rheumatology and Rheumatology Unit, Santiago de Compostela, Spain

Background: Chronic inflammatory immune-mediated diseases, as rheuma toid arthritis (RA), psoriatic arthritis (PsA), psoriasis (Ps), ankylosing spondylitis (AS), ulcerative colitis (UC) and Crohn's disease (CD), have higher risks of morbidity and mortality and a great impact on quality of life of patients ${ }^{1}$. Drug inhibitors of tumor necrosis factor (TNF inhibitors) have demonstrated an adequate efficacy and security profile in these patients when classical treatments (as disease-modifying antirheumatic drugs) have been failed or patients were intolerant. TNF inhibitors are used to contro inflammatory response and to improve quality of life, pain, functional capacity and progression of the disease ${ }^{2}$. Studies in RA show that smoking habit is related to more articular and extra-articular damage, worse prognosis, higher basal activity and higher risk of seropositive RA. It has been related also to greater number of different treatments and higher doses needed for patients by pharmacokinetic and pharmacodynamic processes. Data published until now suggest that smoking habit decreases efficacy of TNF inhibitors ${ }^{3}$.

Objectives: To analyze the smoking habit influence on the efficacy of TNF inhibitors in patients diagnosed of chronic inflammatory immune-mediated diseases (RA, PsA, Ps, AS, UC, CD).

Methods: It was made a systematic literature search using Cochrane Library, Medline, the Web of Science and Embase databases. Meta-analyses were performed using a random-effects model.

Results: 37 of 3677 identified articles met the inclusion criteria. No documents with GOL were found. The analysis of all the diseases together gives a significant decrease on the response to TNF inhibitors in smok ing patients [OR $0.812(0.662-0.996), p=0.046]$. This response also has a significant decrease in IBD maintained response in smoking patients [OR $0.467(0.257-0.848), p=0.012]$. A non-significant decrease in the treatment response of smoking patients with IBD clinical remission was found, in smoking patients versus ex-smoking and never smoking patients with IBD response, in ex-smoking patients versus never smoking patients with IBD partial response, in current and ex-smoking patients versus never smok ing patients with IBD partial response, in current smoking patients versus ex-smoking patients with IBD partial response, in current smoking patients versus never and ex-smoking patients with IBD partial response, in current smoking patients versus never smoking patients with IBD partial response, in patients with $A R$ EULAR and in patients with AR EULAR moderate response.

Conclusion: Smoking habit is a poor prognosis factor in RA, AS, Ps, PsA, UC and CD. Its leaving will decrease cardiovascular risk, joint and bowel damage, will increase the efficacy of TNF inhibitors and will benefit the health of patients, not only in their particular disease, and it can be the first step on their treatment.

\section{REFERENCES}

[1] Gabriel SE. Cardiovascular morbidity and mortality in rheumatoid arthritis Am J Med 2008;121(10):9-14.

[2] Seymour HE. Anti-TNF agents for rheumatoid arthritis. Br J Clin Pharmaco 2001;51(3):201-8

[3] Arnson Y. Effects of tobacco smoke on immunity, inflammation and autoimmunity. J Autoimmun 2010;34(3):258-65.

Disclosure of Interests: None declared

DOI: 10.1136/annrheumdis-2019-eular.1937 